\title{
Invariant Differential Operators for the Real Exceptional Lie Algebra $F_{4}^{\prime}$
}

\author{
V.K. Dobrev* \\ Institute of Nuclear Research and Nuclear Energy, \\ Bulgarian Academy of Sciences, \\ 72 Tsarigradsko Chaussee, 1784 Sofia, Bulgaria \\ E-mail: vkdobrevdvahoo.com
}

In the present paper we continue the project of systematic construction of invariant differential operators on the example of the non-compact exceptional Lie algebra $F_{4}^{\prime}$ which is split real form of the exceptional Lie algebra $F_{4}$. We consider induction from a maximal parabolic algebra. We classify the reducible Verma modules over $F_{4}$ which are compatible with this induction. Thus, we obtain the classification of the corresponding invariant differential operators.

Corfu Summer Institute 2019 "School and Workshops on Elementary Particle Physics and Gravity" (CORFU2019)

31 August - 25 September 2019

Corfù, Greece

${ }^{*}$ Speaker. 


\section{Introduction}

Invariant differential operators play very important role in the description of physical symmetries. The general scheme for constructing these operators was given some time ago [1]. In recent papers $[2,3]$ we started the systematic explicit construction of the invariant differential operators.

The first task in the construction is to make the multiplet classification of the reducible Verma modules over the algebra in consideration following [4]. Such classification provides the weights of embeddings between the Verma modules via the singular vectors, and thus, by [1], the weights of the invariant differential operators.

We have done the multiplet classification for many real non-compact algebras, first from the class of algebras that have discrete series representations, see [5]. In the present paper we focus on the complex exceptional Lie algebra $F_{4}$ and on its split real form algebra $F_{4}^{\prime}$. Our scheme requires that we use induction from parabolic subalgebras. In the present paper we choose a parabolic subalgebra containing the factor $\mathscr{M} \oplus \mathscr{A}$, where $\mathscr{M}=\operatorname{sl}(3, \mathbb{R}) \oplus \operatorname{sl}(2, \mathbb{R}), \mathscr{A}=o(2)$. This choice is motivated by the fact that the complexification of $\mathscr{M} \oplus \mathscr{A}$ and the corresponding compact form $\mathscr{M}_{c} \oplus \mathscr{A}_{c}=s u(3) \oplus s u(2) \oplus u(1)$ have applications in physics being the Lie algebra symmetry of the standard model of elementary particles [6] (see also [7]). ${ }^{1}$

We present the multiplet classification of the reducible Verma modules over $F_{4}$ which are compatible with the chosen parabolic of $F_{4}^{\prime}$. We give also the weights of the singular vectors between these modules. By the scheme of [1] these singular vectors will produce the invariant differential operators.

\section{Preliminaries}

\subsection{Lie algebra}

We start with the complex exceptional Lie algebra $\mathscr{G}^{\mathbb{C}}=F_{4}$. We use the standard definition of $\mathscr{G}^{\mathbb{C}}$ given in terms of the Chevalley generators $X_{i}^{ \pm}, H_{i}, i=1,2,3,4\left(=\operatorname{rank} F_{4}\right)$, by the relations :

$$
\begin{gathered}
{\left[H_{j}, H_{k}\right]=0,\left[H_{j}, X_{k}^{ \pm}\right]= \pm a_{j k} X_{k}^{ \pm},\left[X_{j}^{+}, X_{k}^{-}\right]=\delta_{j k} H_{j},} \\
\sum_{m=0}^{n}(-1)^{m}\left(\begin{array}{c}
n \\
m
\end{array}\right)\left(X_{j}^{ \pm}\right)^{m} X_{k}^{ \pm}\left(X_{j}^{ \pm}\right)^{n-m}=0, j \neq k, n=1-a_{j k},
\end{gathered}
$$

where

$$
\left(a_{i j}\right)=\left(\begin{array}{cccc}
2 & -1 & 0 & 0 \\
-1 & 2 & -1 & 0 \\
0 & -2 & 2 & -1 \\
0 & 0 & -1 & 2
\end{array}\right)
$$

is the Cartan matrix of $\mathscr{G}^{\mathbb{C}}, \alpha_{j}^{\vee} \equiv \frac{2 \alpha_{j}}{\left(\alpha_{j}, \alpha_{j}\right)}$ is the co-root of $\alpha_{j},(\cdot, \cdot)$ is the scalar product of the roots, so that the nonzero products between the simple roots are: $\left(\alpha_{1}, \alpha_{1}\right)=\left(\alpha_{2}, \alpha_{2}\right)=2\left(\alpha_{3}, \alpha_{3}\right)=$ $2\left(\alpha_{4}, \alpha_{4}\right)=2, \quad\left(\alpha_{1}, \alpha_{2}\right)=-1, \quad\left(\alpha_{2}, \alpha_{3}\right)=-1, \quad\left(\alpha_{3}, \alpha_{4}\right)=-1 / 2$. The elements $H_{i}$ span the

\footnotetext{
${ }^{1}$ More precisely the symmetry is presented on the group level as : $G=S U(3) \times S U(2) \times U(1) / Z$, where $Z$ belongs to the center of $G$.
} 
Cartan subalgebra $\mathscr{H}$ of $\mathscr{G}^{\mathbb{C}}$, while the elements $X_{i}^{ \pm}$generate the subalgebras $\mathscr{G}^{ \pm}$. We shall use the standard triangular decomposition

$$
\mathscr{G}^{\mathbb{C}}=\mathscr{G}_{+} \oplus \mathscr{H} \oplus \mathscr{G}_{-}, \quad \mathscr{G}_{ \pm} \equiv \underset{\alpha \in \Delta^{ \pm}}{\bigoplus} \mathscr{G}_{\alpha},
$$

where $\Delta^{+}, \Delta^{-}$, are the sets of positive, negative, roots, resp. Explicitly we have that there are roots of two lengths with length ratio $2: 1$.

The long roots are: $\alpha_{1}, \alpha_{2}, \alpha_{1}+\alpha_{2}, \alpha_{2}+2 \alpha_{3}, \alpha_{1}+\alpha_{2}+2 \alpha_{3}, \alpha_{1}+2 \alpha_{2}+2 \alpha_{3}, \alpha_{2}+2 \alpha_{3}+2 \alpha_{4}$, $\alpha_{1}+\alpha_{2}+2 \alpha_{3}+2 \alpha_{4}, \quad \alpha_{1}+2 \alpha_{2}+2 \alpha_{3}+2 \alpha_{4}, \quad \alpha_{1}+2 \alpha_{2}+4 \alpha_{3}+2 \alpha_{4}, \quad \alpha_{1}+3 \alpha_{2}+4 \alpha_{3}+2 \alpha_{4}$, $2 \alpha_{1}+3 \alpha_{2}+4 \alpha_{3}+2 \alpha_{4}$. With the chosen normalization they have length 2 .

The short roots are: $\alpha_{3}, \alpha_{4}, \alpha_{2}+\alpha_{3}, \alpha_{3}+\alpha_{4}, \alpha_{1}+\alpha_{2}+\alpha_{3}, \alpha_{2}+\alpha_{3}+\alpha_{4}, \alpha_{1}+\alpha_{2}+\alpha_{3}+\alpha_{4}$, $\alpha_{2}+2 \alpha_{3}+\alpha_{4}, \quad \alpha_{1}+2 \alpha_{2}+2 \alpha_{3}+\alpha_{4}, \quad \alpha_{1}+\alpha_{2}+2 \alpha_{3}+\alpha_{4}, \quad \alpha_{1}+2 \alpha_{2}+3 \alpha_{3}+\alpha_{4}, \alpha_{1}+2 \alpha_{2}+$ $3 \alpha_{3}+2 \alpha_{4}$, and they have length 1 .

(Note that the short roots are exactly those which contain $\alpha_{3}$ and/or $\alpha_{4}$ with odd coefficient, while the long roots contain $\alpha_{3}$ and $\alpha_{4}$ with even coefficients.)

Thus, $F_{4}$ is $52-$ dimensional $\left(52=|\Delta|+\operatorname{rank} F_{4}\right)$.

In terms of the normalized basis $\varepsilon_{1}, \varepsilon_{2}, \varepsilon_{3}, \varepsilon_{4}$ we have:

$$
\begin{array}{r}
\Delta^{+}=\left\{\varepsilon_{i}, 1 \leq i \leq 4 ; \varepsilon_{j} \pm \varepsilon_{k}, 1 \leq j<k \leq 4\right. \\
\left.\frac{1}{2}\left(\varepsilon_{1} \pm \varepsilon_{2} \pm \varepsilon_{3} \pm \varepsilon_{4}\right), \text { all signs }\right\}
\end{array}
$$

The simple roots are:

$$
\pi=\left\{\alpha_{1}=\varepsilon_{2}-\varepsilon_{3}, \alpha_{2}=\varepsilon_{3}-\varepsilon_{4}, \alpha_{3}=\varepsilon_{4}, \alpha_{4}=\frac{1}{2}\left(\varepsilon_{1}-\varepsilon_{2}-\varepsilon_{3}-\varepsilon_{4}\right)\right\}
$$

The maximal compact subalgebra is $\mathscr{K}=s p(3) \oplus s u(2)$. Its complexification $\mathscr{K}^{\mathbb{C}}$ may be embedded most easily in $F_{4}$ as the Lie algebra generated by the subalgebras with simple roots $\left\{\alpha_{2}, \alpha_{3}, \alpha_{4}\right\}$ and $\left\{\alpha_{1}\right\}$. The long roots of $s p(3, \mathbb{C})$ in this embedding are: $\alpha_{2}, \alpha_{2}+2 \alpha_{3}$, $\alpha_{2}+2 \alpha_{3}+2 \alpha_{4}$. The short roots are: $\alpha_{3}, \alpha_{4}, \alpha_{2}+\alpha_{3}, \alpha_{3}+\alpha_{4}, \alpha_{2}+\alpha_{3}+\alpha_{4}, \alpha_{2}+2 \alpha_{3}+\alpha_{4}$.

Note that the 16 roots on the first line of (2.4) form the positive root system of $B_{4}$ with simple roots $\varepsilon_{i}-\varepsilon_{i+1}, i=1,2,3, \varepsilon_{4}$.

The Weyl group of $F_{4}$ is the semidirect product of $S_{3}$ with a group which itself is the semidirect product of $S_{4}$ with $(\mathbb{Z} / 2 \mathbb{Z})^{3}$, thus, $|W|=2^{7} 3^{2}=1152$.

\subsection{Verma modules}

Let us recall that a Verma module $V^{\Lambda}$ is defined as the HWM over $\mathscr{G}^{\mathbb{C}}$ with highest weight $\Lambda \in \mathscr{H}^{*}$ and highest weight vector $v_{0} \in V^{\Lambda}$, induced from the one-dimensional representation $V_{0} \cong \mathbb{C} v_{0}$ of $U(\mathscr{B})$, where $\mathscr{B}=\mathscr{H} \oplus \mathscr{G}_{+}$is a Borel subalgebra of $\mathscr{G} \mathbb{C}$, such that:

$$
\begin{aligned}
& X v_{0}=0, \quad \forall X \in \mathscr{G}_{+} \\
& H v_{0}=\Lambda(H) v_{0}, \quad \forall H \in \mathscr{H}
\end{aligned}
$$

Verma modules are generically irreducible. A Verma module $V^{\Lambda}$ is reducible [8] iff there exists a root $\beta \in \Delta^{+}$and $m \in \mathbb{N}$ such that

$$
\left(\Lambda+\rho, \beta^{\vee}\right)=m
$$


holds, where $\rho=\frac{1}{2} \sum_{\alpha \in \Delta^{+}} \alpha,\left(\rho=8 \alpha_{1}+15 \alpha_{2}+21 \alpha_{3}+11 \alpha_{4}\right)$.

If (2.7) holds then the reducible Verma module $V^{\Lambda}$ contains an invariant submodule which is also a Verma module $V^{\Lambda^{\prime}}$ with shifted weight $\Lambda^{\prime}=\Lambda-m \beta$. This statement is equivalent to the fact that $V^{\Lambda}$ contains a singular vector $v_{s} \in V^{\Lambda}$, such that $v_{s} \neq \xi v_{0},(0 \neq \xi \in \mathbb{C})$, and :

$$
\begin{aligned}
& X v_{s}=0, \quad \forall X \in \mathscr{G}_{+} \\
& H v_{s}=\Lambda^{\prime}(H) v_{s}, \quad \Lambda^{\prime}=\Lambda-m \beta, \quad \forall H \in \mathscr{H}
\end{aligned}
$$

More explicitly, [1],

$$
v_{m, \beta}^{s}=\mathscr{P}_{m, \beta} v_{0} \text {. }
$$

The general reducibility conditions ([R.] for $V^{\Lambda}$ spelled out for the simple roots in our situation are:

$$
\begin{aligned}
& m_{1} \equiv m_{\alpha_{1}}=\left(\Lambda+\rho, \alpha_{1}\right), \quad m_{2} \equiv m_{\alpha_{2}}=\left(\Lambda+\rho, \alpha_{2}\right), \\
& m_{3} \equiv m_{\alpha_{3}}=\left(\Lambda+\rho, 2 \alpha_{3}\right), \quad m_{4} \equiv m_{\alpha_{4}}=\left(\Lambda+\rho, 2 \alpha_{4}\right)
\end{aligned}
$$

The numbers $m_{i}$ from (2.10) corresponding to the simple roots are called Dynkin labels, while the more general Harish-Chandra parameters are:

$$
m_{\beta}=\left(\Lambda+\rho, \beta^{\vee}\right), \quad \beta \in \Delta^{+}
$$

\subsection{Structure theory of the real form}

The split real form of $F_{4}$ is denoted as $F_{4}^{\prime}$, sometimes as $F_{4(4)}$. This real form has discrete series representations since $\operatorname{rank} F_{4}^{\prime}=\operatorname{rank} \mathscr{K}$. We can use the same basis (but over $\mathbb{R}$ ) and the same root system.

The Iwasawa decomposition of the real split form $\mathscr{G} \equiv F_{4}^{\prime}$, is:

$$
\mathscr{G}=\mathscr{K} \oplus \mathscr{A}_{0} \oplus \mathscr{N}_{0}
$$

the Cartan decomposition is:

$$
\mathscr{G}=\mathscr{K} \oplus \mathscr{Q},
$$

where we use: the maximal compact subgroup $\mathscr{K} \cong s p(3) \oplus s u(2), \operatorname{dim}_{\mathbb{R}} \mathscr{Q}=28, \operatorname{dim}_{\mathbb{R}} \mathscr{A}_{0}=4$, $\mathscr{N}_{0}=\mathscr{N}_{0}^{+}$, or $\mathscr{N}_{0}=\mathscr{N}_{0}^{-} \cong \mathscr{N}_{0}^{+}, \operatorname{dim}_{\mathbb{R}} \mathscr{N}_{0}^{ \pm}=24$.

Since $\mathscr{G}$ is maximally split, then the centralizer $\mathscr{M}_{0}$ of $\mathscr{A}_{0}$ in $\mathscr{K}$ is zero, thus, the minimal parabolic $\mathscr{P}_{0}$ and the corresponding Bruhat decomposition are:

$$
\mathscr{P}_{0}=\mathscr{A}_{0} \oplus \mathscr{N}_{0}, \quad \mathscr{G}=\mathscr{A}_{0} \oplus \mathscr{N}_{0}^{+} \oplus \mathscr{N}_{0}^{-}
$$

The importance of the parabolic subgroups comes from the fact that the representations induced from them generate all (admissible) irreducible representations of the group under consideration [9-11].

We recall that in general a parabolic subalgebra $\mathscr{P}=\mathscr{M} \oplus \mathscr{A} \oplus \mathscr{N}$ is any subalgebra of $\mathscr{G}$ which contains a minimal parabolic subalgebra $\mathscr{M}_{0}$. In general, $\mathscr{M}$ contains the subalgebra $\mathscr{M}_{0}$, while $\mathscr{A}$ is contained in $\mathscr{A}_{0}, \mathscr{N}$ is contained in $\mathscr{N}_{0}$.

On the other extreme are the maximal parabolic subalgebras for which $\operatorname{dim} \mathscr{A}=1$. 


\subsection{Elementary representations}

Further, let $G, K, P, M, A, N$ are Lie groups with Lie algebras $\mathscr{G}_{0}, \mathscr{K}, \mathscr{P}, \mathscr{M}, \mathscr{A}, \mathscr{N}$.

Let $v$ be a (non-unitary) character of $A, v \in \mathscr{A}^{*}$. Let $\mu$ fix a finite-dimensional (nonunitary) representation $D^{\mu}$ of $M$ on the space $V_{\mu}$. In the case when $M$ is cuspidal then we may use also the discrete series representation of $M$ with the same Casimirs as $D^{\mu}$. (We ignore a possible discrete center of $M$ since its representations are not relevant for the construction of invariant differential operators [5].)

We call the induced representation $\chi=\operatorname{Ind}_{P}^{G}(\mu \otimes \boldsymbol{v} \otimes 1)$ an elementary representation of $G$ [12]. (These are called generalized principal series representations (or limits thereof) in [13].) Their spaces of functions are:

$$
\mathscr{C}_{\chi}=\left\{\mathscr{F} \in C^{\infty}\left(G, V_{\mu}\right) \mid \mathscr{F}(\text { gman })=e^{-v(H)} \cdot D^{\mu}\left(m^{-1}\right) \mathscr{F}(g)\right\}
$$

where $a=\exp (H) \in A, H \in \mathscr{A}, m \in M, n \in N$. The representation action is the left regular action:

$$
\left(\mathscr{T}^{\chi}(g) \mathscr{F}\right)\left(g^{\prime}\right)=\mathscr{F}\left(g^{-1} g^{\prime}\right), \quad g, g^{\prime} \in G .
$$

An important ingredient in our considerations are the highest/lowest weight representations of $\mathscr{G}$. These can be realized as (factor-modules of) Verma modules $V^{\Lambda}$ over $\mathscr{G}$, where $\Lambda \in(\mathscr{H})^{*}$, the weight $\Lambda=\Lambda(\chi)$ being determined uniquely from $\chi$ [1].

As we have seen when a Verma module is reducible and (2.7) holds then there is a singular vector (2.9). Relatedly, then there exists [1] an invariant differential operator

$$
\mathscr{D}_{m, \beta}: \mathscr{C}_{\chi(\Lambda)} \longrightarrow \mathscr{C}_{\chi(\Lambda-m \beta)}
$$

given explicitly by:

$$
\mathscr{D}_{m, \beta}=\mathscr{P}_{m, \beta}\left(\widehat{\mathscr{N}^{-}}\right)
$$

where $\widehat{\mathscr{N}^{-}}$denotes the right action on the functions $\mathscr{F}$.

Actually, since our ERs are induced from finite-dimensional representations of $\mathscr{M}$ the corresponding Verma modules are always reducible. Thus, it is more convenient to use generalised Verma modules $\tilde{V}^{\Lambda}$ such that the role of the highest/lowest weight vector $v_{0}$ is taken by the (finitedimensional) space $V_{\mu} v_{0}$.

Algebraically, the above is governed by the notion of $\mathscr{M}$-compact roots of $\mathscr{G} \mathbb{C}$. These are the roots of $\mathscr{G}^{\mathbb{C}}$ which can be identified as roots of $\mathscr{M}^{\mathbb{C}}$ as the latter is a subalgebra of $\mathscr{G}^{\mathbb{C}}$. The consequence of this is that $(\mathbb{Z} \mathbb{Z})$ is always fulfilled for the $\mathscr{M}$-compact roots of $\mathscr{G} \mathbb{C}$. That is why we consider generalised Verma modules. Relatedly, the invariant differential operators corresponding to $\mathscr{M}$-compact roots are trivial.

\section{Invariant differential operators for $F_{4}^{\prime}$}

The real form $F_{4}^{\prime}$ has several parabolic subalgebras [2]. We shall consider the maximal parabolic subalgebra [2]:

$$
\begin{aligned}
& \mathscr{P}=\mathscr{M} \oplus \mathscr{A} \oplus \mathscr{N}, \\
& \mathscr{M}=\operatorname{sl}(3, \mathbb{R}) \oplus(2, \mathbb{R}), \\
& \operatorname{dim} \mathscr{A}=1, \quad \operatorname{dim} \mathscr{N}=20
\end{aligned}
$$


such that the embedding of $\mathscr{M}$ and $\mathscr{M}^{\mathbb{C}}$ in $\mathscr{G}^{\mathbb{C}}$ is given by:

$$
\operatorname{sl}(3, \mathbb{R})^{\mathbb{C}}:\left\{\alpha_{1}, \alpha_{2}, \alpha_{12}=\alpha_{1}+\alpha_{2}\right\}, \quad \operatorname{sl}(2, \mathbb{R})^{\mathbb{C}}:\left\{\alpha_{4}\right\}
$$

Remark: Note that $F_{4}^{\prime}$ has a another maximal parabolic subalgebra that is also written as (B.D) but the embedding of $\mathscr{M}$ and $\mathscr{M}^{\mathbb{C}}$ flips the short and long roots [2]:

$$
s l(3, \mathbb{R})^{\mathbb{C}}:\left\{\alpha_{3}, \alpha_{4}, \alpha_{34}=\alpha_{3}+\alpha_{4}\right\}, \quad \operatorname{sl}(2, \mathbb{R})^{\mathbb{C}}:\left\{\alpha_{1}\right\}
$$

That case is also very interesting and will be considered next [14].

Further we classify the generalized Verma modules (GVM) relative to the maximal parabolic subalgebra (B.]). This also provides the classification of the $P$-induced ERs with the same Casimirs. The classification is done as follows. We group the reducible Verma modules (also the corresponding ERs) related by nontrivial embeddings in sets called multiplets [1,4]. These multiplets may be depicted as a connected graph, the vertices of which correspond to the GVMs and the lines between the vertices correspond to the GVM embeddings (and also the invariant differential operators between the ERs). The explicit parametrization of the multiplets and of their Verma modules (and ERs) is important for understanding of the situation.

The result of our classification is a follows. The multiplets of GVMs (and ERs) induced from (B.]) are parametrized by four positive integers $\chi=\left[m_{1}, m_{2}, m_{3}, m_{4}\right]$. Each multiplet contains 96 GVMs (ERs). They are presented in Fig. 1.

On the figure each arrow represents an embedding between two Verma modules, $V^{\Lambda}$ and $V^{\Lambda^{\prime}}$, the arrow pointing to the embedded module $V^{\Lambda^{\prime}}$. Each arrow carries a number $n, n=1,2,3,4$, which indicates the level of the embedding, $\Lambda^{\prime}=\Lambda-m_{n} \beta$ [5]. Another feature is indicated by the enumeration of the GVMs (ERs). Namely, if $\Lambda$ corresponds to signature $\chi_{k, \ell}^{-}, k<9$, then $\Lambda^{\prime}$ corresponds to signature $\chi_{k+1, \ell^{\prime}}^{-}$(where $\ell, \ell^{\prime}$ are secondary enumerations that are absent in some cases). Analogously: if $\Lambda$ corresponds to signature $\chi_{k, \ell}^{+}, k>10$, then $\Lambda^{\prime}$ corresponds to signature $\chi_{k-1, \ell^{\prime}}^{+}$; if $\Lambda$ corresponds to signature $\chi_{9, \ell}^{-}$, then $\Lambda^{\prime}$ corresponds to signature $\chi_{10, \ell^{\prime}}^{*}$, (where $*$ may happen to $\mathrm{be}^{\prime}+^{\prime}$ or $^{\prime}-^{\prime}$ ); if $\Lambda$ corresponds to signature $\chi_{10, \ell}^{*}$, then $\Lambda^{\prime}$ corresponds to signature $\chi_{9, \ell^{\prime}}^{+}$.

Further, we can see there is an additional symmetry. It is relevant for the ERs and indicates the integral intertwining Knapp-Stein (KS) operators acting between the ERs. Due to this symmetry in the actual parametrization we shall use the conformal weight $d=7 / 2+c$, more precisely, the parameter $c$, instead of the non-compact Dynkin label $m_{3}$. The parameter $c$ is more convenient since the KS operators flip its sign. The KS operators also involve an $\operatorname{sl}(3)$ flip of the Dynkin labels $m_{1}, m_{2}$ (see below). Thus, the entries are:

$$
\chi=\left\{n_{1}, n_{2}, c, n_{4}\right\}
$$

so that for the top ER (GVM) on the figure $\Lambda_{0}^{-}$we have:

$$
\chi_{0}^{-}=\left\{n_{1}=m_{1}, n_{2}=m_{2}, c=-\left(m_{1}+m_{2}+m_{3}+m_{4} / 2\right), n_{4}=m_{4}\right\}
$$

Furthermore the $\operatorname{sl}(3)$ flip $\left(n_{1}, n_{2}\right)^{ \pm}$will be given below by:

$$
\left(n_{1}, n_{2}\right)^{+}=\left(n_{1}, n_{2}\right), \quad\left(n_{1}, n_{2}\right)^{-}=\left(n_{2}, n_{1}\right)
$$


Altogether, the explicit parametrization of the multiplets is given by:

$$
\begin{aligned}
& \chi_{0}^{\mp}=\left\{\left(m_{1}, m_{2}\right)^{ \pm}, \mp\left(m_{1}+m_{2}+m_{3}+m_{4} / 2\right), m_{4}\right\}, \\
& \chi_{1}^{\mp}=\left\{\left(m_{1}, m_{23}\right)^{ \pm}, \mp\left(m_{12}+m_{34} / 2\right), m_{34}\right\} \text {, } \\
& \chi_{2,1}^{\mp}=\left\{\left(m_{12}, m_{23}\right)^{ \pm}, \mp\left(m_{12}+m_{34} / 2\right), m_{24}+m_{2}\right\}, \\
& \chi_{2,2}^{\mp}=\left\{\left(m_{1}, m_{24}\right)^{ \pm}, \mp\left(m_{12}+m_{3} / 2\right), m_{3}\right\} \text {, } \\
& \chi_{3}^{\mp}=\left\{\left(m_{12}, m_{24}\right)^{ \pm}, \mp\left(m_{12}+m_{3} / 2\right), m_{23}+m_{2}\right\} \text {, } \\
& \chi_{3,1}^{\mp}=\left\{\left(m_{2}, m_{13}\right)^{ \pm}, \mp\left(m_{2}+m_{34} / 2, m_{14}+m_{12}\right\}\right. \text {, } \\
& \chi_{3,2}^{\mp}=\left\{\left(m_{13}, m_{2}\right)^{ \pm}, \mp\left(m_{13}+m_{4} / 2\right), m_{24}+m_{23}\right\} \text {, } \\
& \chi_{4,1}^{\mp}=\left\{\left(m_{14}, m_{2}\right)^{ \pm}, \mp\left(m_{2}+m_{3} / 2\right), m_{13}+m_{12}\right\}, \\
& \chi_{4,2}^{\mp}=\left\{\left(m_{13}, m_{24}\right)^{ \pm}, \mp\left(m_{12}+m_{3} / 2\right), m_{2}+m_{23}\right\}, \\
& \chi_{4,3}^{\mp}=\left\{\left(m_{23}, m_{12}^{ \pm}, \mp\left(m_{23}+m_{4} / 2\right), m_{14}+m_{13}\right\}\right. \text {, } \\
& \chi_{4,4}^{\mp}=\left\{\left(m_{14}, m_{2}\right)^{ \pm}, \mp\left(m_{13}+m_{4} / 2\right), m_{24}+m_{23}\right\} \text {, } \\
& \chi_{5}^{\mp}=\left\{\left(m_{23}, m_{14}\right)^{ \pm}, \mp\left(m_{2}+m_{3} / 2\right), m_{13}+m_{12}\right\}, \\
& \chi_{5,1}^{\mp}=\left\{\left(m_{13}+m_{2}, m_{24}\right)^{ \pm}, \mp\left(m_{12}+m_{3} / 2\right), m_{3}\right\} \text {, } \\
& \chi_{5,2}^{\mp}=\left\{\left(m_{14}, m_{23}\right)^{ \pm}, \mp\left(m_{12}+m_{34} / 2\right), m_{24}+m_{2}\right\}, \\
& \chi_{5,3}^{\mp}=\left\{\left(m_{24}, m_{12}\right)^{ \pm}, \mp\left(m_{23}+m_{4} / 2\right), m_{14}+m_{13}\right\}, \\
& \chi_{5,4}^{\mp}=\left\{\left(m_{23}, m_{1}\right)^{ \pm}, \mp\left(m_{23}+m_{4} / 2\right), m_{14}+m_{13}+2 m_{2}\right\}, \\
& \chi_{6,1}^{\mp}=\left\{\left(m_{23}, m_{14}+m_{2}\right)^{ \pm}, \mp\left(m_{3} / 2\right), m_{13}+m_{12}\right\}, \\
& \chi_{6,2}^{\mp}=\left\{\left(m_{24}, m_{13}\right)^{ \pm}, \mp\left(m_{2}+m_{34} / 2\right), m_{14}+m_{12}\right\}, \\
& \chi_{6,3}^{\mp}=\left\{\left(m_{14+m_{2}}, m_{23}\right)^{ \pm}, \mp\left(m_{12}+m_{34} / 2\right), m_{34}\right\}, \\
& \chi_{6,4}^{\mp}=\left\{\left(m_{13}+m_{2}, m_{14}\right)^{ \pm}, \mp\left(m_{2}+m_{3} / 2\right), m_{3}\right\}, \\
& \chi_{6,5}^{\mp}=\left\{\left(m_{24}, m_{13}\right)^{ \pm}, \mp\left(m_{23}+m_{4} / 2\right), m_{14}+m_{13}+2 m_{2}\right\} \text {, } \\
& \chi_{6,6}^{\mp}=\left\{\left(m_{24}, m_{13}\right)^{ \pm}, \mp\left(m_{2}+m_{34} / 2\right), m_{14}+m_{13}+m_{23}+m_{2}\right\}, \\
& \chi_{7}^{\mp}=\left\{\left(m_{24}, m_{13}+m_{2}\right)^{ \pm}, \mp\left(m_{34} / 2\right), m_{14}+m_{12}\right\}, \\
& \chi_{7,1}^{\mp}=\left\{\left(m_{13}, m_{14}+m_{2}\right)^{ \pm}, \mp\left(m_{3} / 2\right), m_{23}+m_{2}\right\} \text {, } \\
& \chi_{7,2}^{\mp}=\left\{\left(m_{2}, m_{14}+m_{23}\right)^{ \pm}, \pm\left(m_{3} / 2\right), m_{13}+m_{12}\right\}, \\
& \chi_{7,3}^{\mp}=\left\{\left(m_{24}, m_{1}\right)^{ \pm}, \mp\left(m_{2}+m_{34} / 2\right), m_{14}+m_{13}+m_{23}+m_{2}\right\}, \\
& \chi_{7,4}^{\mp}=\left\{\left(m_{14}+m_{23}, m_{2}\right)^{ \pm}, \mp\left(m_{13}+m_{4} / 2\right), m_{4}\right\} \text {, } \\
& \chi_{7,5}^{\mp}=\left\{\left(m_{14}+m_{2}, m_{13}\right)^{ \pm}, \mp\left(m_{2}+m_{34} / 2\right), m_{34}\right\}, \\
& \chi_{7,6}^{\mp}=\left\{\left(m_{2}, m_{1}\right)^{ \pm}, \mp\left(m_{2}+m_{34} / 2\right), 2 m_{14}+m_{23}+m_{2}\right\}, \\
& \chi_{8,1}^{\mp}=\left\{\left(m_{24}, m_{13}+m_{2}\right)^{ \pm}, \mp\left(m_{4} / 2\right), m_{13}+m_{14}\right\} \text {, } \\
& \chi_{8,2}^{\mp}=\left\{\left(m_{14}, m_{13}+m_{2}\right)^{ \pm}, \mp\left(m_{34} / 2\right), m_{24}+m_{2}\right\}, \\
& \chi_{8,3}^{\mp}=\left\{\left(m_{24}, m_{12}\right)^{ \pm}, \mp\left(m_{34} / 2\right), m_{14}+m_{13}+m_{23}+m_{2}\right\}, \\
& \chi_{8,4}^{\mp}=\left\{\left(m_{23}, m_{1}\right)^{ \pm}, \mp\left(m_{2}+m_{3} / 2\right), 2 m_{14}+m_{23}+m_{2}\right\}, \\
& \chi_{8,5}^{\mp}=\left\{\left(m_{14}+m_{23}, m_{12}\right)^{ \pm}, \mp\left(m_{23}+m_{4} / 2\right), m_{4}\right\} \text {, } \\
& \chi_{8,6}^{\mp}=\left\{\left(m_{2}, m_{14}+m_{23}\right)^{ \pm}, \pm m_{34} / 2, m_{14}+m_{12}\right\} \text {, } \\
& \chi_{8,7}^{\mp}=\left\{\left(m_{12}, m_{14}+m_{23}\right)^{ \pm}, \pm m_{3} / 2, m_{23}+m_{2}\right\} \text {, }
\end{aligned}
$$




$$
\begin{aligned}
& \chi_{9}^{\mp}=\left\{\left(m_{14}, m_{13}+m_{2}\right)^{ \pm}, \mp\left(m_{4} / 2\right), m_{24}+m_{23}\right\}, \\
& \chi_{9,1}^{\mp}=\left\{\left(m_{24}, m_{13}\right)^{ \pm}, \mp\left(m_{4} / 2\right), 2 m_{14}+m_{23}+m_{2}\right\}, \\
& \chi_{9,2}^{\mp}=\left\{\left(m_{14}+m_{23}+m_{2}, m_{1}\right)^{ \pm}, \mp\left(m_{23}+m_{4} / 2\right), m_{4}\right\}, \\
& \chi_{9,3}^{\mp}=\left\{\left(m_{23}, m_{14}+m_{2}\right)^{ \pm}, \pm m_{4} / 2, m_{14}+m_{13}\right\}, \\
& \chi_{9,4}^{\mp}=\left\{\left(m_{14}, m_{2}\right)^{ \pm}, \mp\left(m_{34} / 2\right), m_{14}+m_{13}+m_{23}+m_{2}\right\}, \\
& \chi_{9,5}^{\mp}=\left\{\left(m_{1}, m_{14}+m_{23}+m_{2}\right)^{ \pm}, \pm\left(m_{12}+m_{3} / 2\right), m_{3}\right\}, \\
& \chi_{9,6}^{\mp}=\left\{\left(m_{12}, m_{14}+m_{23}\right)^{ \pm}, \pm m_{34} / 2, m_{24}+m_{2}\right\}, \\
& \chi_{9,7}^{\mp}=\left\{\left(m_{23}, m_{12}\right)^{ \pm}, \mp\left(m_{3} / 2\right), 2 m_{14}+m_{23}+m_{2}\right\}, \\
& \chi_{10,1}^{\mp}=\left\{\left(m_{14}+m_{2}, m_{13}\right)^{ \pm}, \mp m_{4} / 2, m_{23}+m_{24}\right\}, \\
& \chi_{10,2}^{\mp}=\left\{\left(m_{13}, m_{2}\right)^{ \pm}, \mp m_{3} / 2,2 m_{14}+m_{23}+m_{2}\right\}, \\
& \chi_{10,3}^{\mp}=\left\{\left(m_{14}, m_{23}\right)^{ \pm}, \mp m_{4} / 2, m_{14}+m_{13}+2 m_{2}\right\}, \\
& \chi_{10,4}^{\mp}=\left\{\left(m_{1}, m_{14}+m_{23}+m_{2}\right)^{ \pm}, \pm\left(m_{2}+m_{34} / 2\right), m_{34}\right\}
\end{aligned}
$$

The pairs $\Lambda^{ \pm}\left(\chi^{ \pm}\right)$are symmetrically placed w.r.t. to the bullet in the middle of the figure.

\section{Concluding remarks}

\section{Remark 1:}

The integral intertwining KS operators act between the spaces $\mathscr{C}_{\chi^{\mp}}$ in opposite directions:

$$
G_{K S}^{+}: \mathscr{C}_{\chi^{-}} \longrightarrow \mathscr{C}_{\chi^{+}}, \quad G_{K S}^{-}: \mathscr{C}_{\chi^{+}} \longrightarrow \mathscr{C}_{\chi^{-}}
$$

\section{Remark 2:}

The positive integers $\left\{m_{1}, m_{2}, m_{3}, m_{4}\right\}$ parametrize the finite-dimensional nonunitary irreps of $F_{4}^{\prime}$ (also the unitary finite-dimensional irreps of the compact Lie algebra $f_{4}$ ).

\section{Remark 3:}

We expect that the discrete series are contained in the representation $\chi_{0}^{+}$since it is dual to $\chi_{0}^{-}$where are contained the finite-dimensional (non-unitary) irreps. Following the Harish-Chandra criterion we must check which $\mathscr{M}$-non-compact entries are negative. We recall that the $\mathscr{M}$ compact entries are $m_{1}^{\prime}, m_{2}^{\prime}, m_{12}^{\prime}, m_{4}^{\prime}$, all other are non-compact. It is easy to see that all the $\mathscr{M}$ non-compact entries are negative. The discrete series irrep with lowest possible conformal weight $d=7$ happens naturally when $m_{1}=m_{2}=m_{3}=m_{4}=1$. It corresponds to the one-dimensional irrep contained in $\chi_{0}^{-}$.

Acknowledgments. The author has received partial support from Bulgarian NSF Grant DN-18/1. 


\section{References}

[1] V.K. Dobrev, Rept. Math. Phys. 25, 159-181 (1988) ; first as ICTP Trieste preprint IC/86/393 (1986).

[2] V.K. Dobrev, Rev. Math. Phys. 20 (2008) 407-449; hep-th/0702152.

[3] V.K. Dobrev, J. High Energy Phys. 02 (2013) 015, arXiv:1208.0409.

[4] V.K. Dobrev, Lett. Math. Phys. 9, 205-211 (1985).

[5] Vladimir K. Dobrev, Invariant Differential Operators, Volume 1: Noncompact Semisimple Lie Algebras and Groups, De Gruyter Studies in Mathematical Physics, vol. 35 (De Gruyter, Berlin, Boston, 2016).

[6] S. Weinberg, The quantum theory of fields (vol 2) (Cambridge University Press, 1996).

[7] M. Dubois-Violette and I. Todorov, Nucl. Phys. B938 (2019) 751-761, arXiv:1808.08110 [hep-th].

[8] I.N. Bernstein, I.M. Gel'fand, S.I. Gel'fand, "Structure of representations generated by highest weight vectors", Funkts. Anal. Prilozh. 5(1) 1-9(1971); English translation: Funct. Anal. Appl. 5 1-8 (1971).

[9] R.P. Langlands, On the classification of irreducible representations of real algebraic groups, Math. Surveys and Monographs, Vol. 31 (AMS, 1988), first as IAS Princeton preprint (1973).

[10] D.P. Zhelobenko, Harmonic Analysis on Semisimple Complex Lie Groups, (Moscow, Nauka, 1974, in Russian).

[11] A.W. Knapp and G.J. Zuckerman, "Classification theorems for representations of semisimple groups", in: Lecture Notes in Math., Vol. 587 (Springer, Berlin, 1977) pp. 138-159;

[12] V.K. Dobrev, G. Mack, V.B. Petkova, S.G. Petrova and I.T. Todorov, Harmonic Analysis on the n-Dimensional Lorentz Group and Its Applications to Conformal Quantum Field Theory, Lecture Notes in Physics, No 63, 280 pages (Springer Verlag, Berlin-Heidelberg-New York, 1977).

[13] A.W. Knapp, Representation Theory of Semisimple Groups (An Overview Based on Examples.), (Princeton Univ. Press 1986).

[14] V.K. Dobrev, in preparation. 


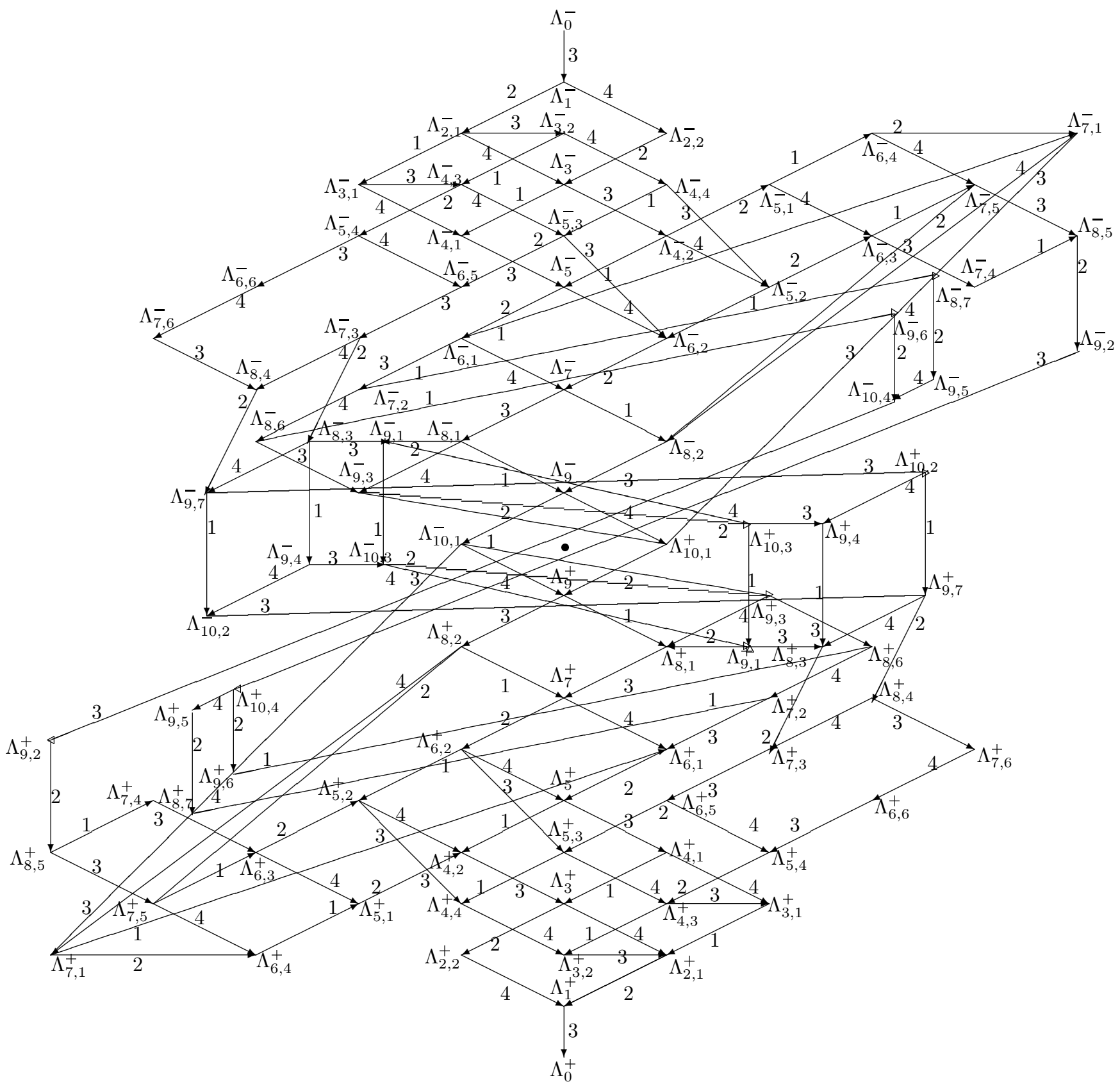

Fig. 1. Multiplets for the real split form $F_{4}^{\prime}$ using maximal parabolic with $\mathcal{M}=\operatorname{sl}(3, \mathbb{R})_{\text {long roots }} \oplus s l(2, \mathbb{R})_{\text {short roots }}$ 